\title{
Mechanical and sensorineural vestibular dysfunction: a case study of "mixed" vestibulopathy
}

\begin{abstract}
A diagnosis of hearing loss typically consists of categorizing the locus of involvement; conductive, sensorineural, retrocochlear, or mixed. It is this designation that provides guidance as to whether medical or a non-medical approach e.g. amplification is the preferred treatment. When a "mixed" loss occurs, a combination of medical and nonmedical management strategies will be utilized. When vestibular dysfunction, is discussed, however, we rarely think about this combined causation. A unilateral vestibulopathy dysfunction (UVD) from a vestibular neuritis, may cause either hair cell damage and/or a neuropathy. This may be considered as a "sensorineural" dysfunction. The literature, and clinical experience, suggests, that an onset of Benign Paroxysmal Positional Vertigo (BPPV) often follows, within days or weeks. BPPV may be considered the "mechanical" component, requiring treatment with Canalith Repositioning. Such patients, therefore, may be considered to have a "mixed" dysfunction. This requires two different treatments in order to successfully extinguish or ameliorate two differing sets of functional impairments. We present a case report of a 52-year-old female patient with complaints of initial acute onset vertigo, positional dizziness, oscillopsia and visual provocation. Attending physician, suspected BPPV and referred her for testing. The evaluation confirmed a left ear BPPV$\mathrm{PC}$, in addition to a non-compensated left unilateral vestibular dysfunction (UVD). The BPPV was successfully cleared with Canalith Repositioning Maneuvers (CRM) at her first treatment visit. Based on her profile ensuring safety and no fall-risk, she was prescribed an individualized self-directed vestibular rehabilitation therapy home exercise program to address the non-BPPV related symptoms. She returned to The American Institute of Balance (AIB), at one-month, reporting complete amelioration of the movement and visually provoked symptoms. Post-therapy objective testing demonstrated compensation of the previously identified UVD. This case demonstrates, that for many patients presenting with BPPV symptoms, there may also be compounding underlying functional impairments. Without the benefit of a thorough evaluation, the "sensorineural" aspect of the "mixed" vestibular dysfunction, may go unresolved. This will further delay their recovery and return to a normal active lifestyle or employment.
\end{abstract}

Keywords: vestibulopathy, benign paroxysmal positional vertigo, canalith repositioning, vestibular rehabilitation therapy
Volume 12 Issue 3 - 2020

\author{
Joseph T Sakumura, Richard E Gans \\ The American Institute of Balance, USA
}

Correspondence: Richard E Gans, Founder \& CEO, The American Institute of Balance, USA, Tel 727.686.4622, Fax 727.398.49I4, Email rgans@dizzy.com

Received: May 14, 2020 | Published: May 26, 2020
Abbreviations: BPPV, benign paroxysmal positional vertigo; UVD, unilateral vestibular dysfunction; $m$ Hallpike, modified hallpike; CDP, computerized dynamic posturography; VRT, vestibular rehabilitation therapy; AIB-CDVAT, AIB-computerized dynamic visual acuity test; cVEMP, cervical vestibular evoked myogenic potentials; VNG, video-nystagmography; UVD, unilateral vestibular dysfunction

\section{Introduction}

Dizziness and vertigo are two of the most common complaints of individuals to primary care physicians throughout the lifespan. Further these symptoms are estimated to account for $5 \%$ of walk-in clinic and $4 \%$ of emergency department (ED) visits. ${ }^{1}$ Benign paroxysmal positional vertigo (BPPV) is the most common otologic cause of vertigo. The condition occurs as a result of mechanical movement of otolithic debris, which become displaced from the macula of the utricle into one of three semi-circular canals. This mechanical displacement causes abnormal stimulation of the velocity sensing canal rendering it responsive to changes in gravity-inertial vector. Its mechanical nature makes BPPV highly responsive to canalith repositioning maneuvers, which reposition displaced debris back into the utricle. These maneuvers are highly efficacious with the literature supporting $90 \%$ success rate of clearance after just 1.25 treatments. ${ }^{2}$
While in most cases BPPV is idiopathic, this condition may also occur secondary to either otologic disease or disorder (e.g. vestibular neuritis) or head trauma and labyrinthine concussion. ${ }^{3}$ While BPPV itself is simply a benign mechanical abnormality, the morbidities that may accompany or precede onset of BPPV have the potential to cause damage to central or peripheral vestibular mechanisms, leading to sensorineural vestibulopathy. Symptoms of vestibulopathy may include; vertigo, postural instability, oscillopsia, and vestibular recruitment. All of which may also be reported by patients with only BPPV. Therefore, it may be difficult for the healthcare practitioner to differentiate between BPPV (mechanical) and vestibulopathy (sensorineural) based on symptoms alone. Furthermore, treatment intervention for BPPV and sensorineural vestibulopathy differ significantly. The most effective vestibular rehabilitation therapy (VRT) is carried out utilizing diagnosis-based strategies and is predicated on comprehensive evaluation determining any existing mechanical and/or sensorineural dysfunction. We therefore opine that most patients, even those with BPPV symptoms, would benefit from a comprehensive evaluation, to provide the best clinical pathway for management. Pre and post VRT test results, also provide objective measures allowing for the patient's return to work or everyday activities. We present such a case with the initial evaluation data and post-VRT findings. 


\section{Case history}

A 52-year-old female patient presented to the AIB clinic with the primary complaints of vertigo, dizziness and imbalance. Symptoms are described as constant "wooziness" and feeling as if her eyes cannot stabilize when she moves her head. She also describes short episodes of room spinning when she lies down or looks up. Symptom onset was about two months ago, at which time she reports an initial attack of vertigo that lasted "almost the entire day". The episode was accompanied by nausea and persistent vomiting. She presented to the Emergency Department, was hospitalized with overnight stay and underwent both CT and MRI scans, which were unremarkable. Her initial diagnosis was "vertigo", and she was prescribed meclizine. She was provided a printed copy of Cawthorne-Cooksey exercises to do at home, without a demonstration or full explanation. She performed these for a week, they did make her dizzy, but have not alleviated any of the symptoms. This initial attack was not preceded by any head trauma or medication change although she does report a mild shingles outbreak in the weeks prior to the episode. After the episode she is left with residual persistent dizziness and imbalance which are exacerbated by head movement, particularly in the horizontal plane. She reports symptoms of oscillopsia and worsening of dizziness with head rotation. While she notes gradual slight improvement in overall dizziness since the initial attack two months ago, she feels as if she cannot move without feeling dizzy and is thus guarded in her movements. Her functional impairment is such that she cannot perform her occupation as a veterinarian assistant effectively. Further, she has concerns that she may have another attack. She reports that she is not sleeping well when laying on her back or changing position, because of the intense, but short-lived vertigo. She denies any recent hearing changes or other otologic complaints currently and medical history is reportedly unremarkable.

\section{Results and analysis}

\section{Audiologic evaluation}

Audiometric testing was performed. All tests including; immittance, otoacoustic emissions, pure tone thresholds, and speech testing were all within normal limits.

\section{Computerized dynamic posturography (CDP)}

As shown, in Fig.1. the sensory organization test (SOT) at initial evaluation revealed sway deviation and a pattern of falls in the vision denied condition on dynamic surface. Evaluation of the vestibulospinal reflex (VSR) yielded a pattern of postural instability consistent with vestibular dysfunction. Motor Control and Adaptation subtests were within age and height normalized parameters. Also shown in Figure 1 are results post treatment which reveal normalized pattern of postural stability indicative of compensation of the VSR.
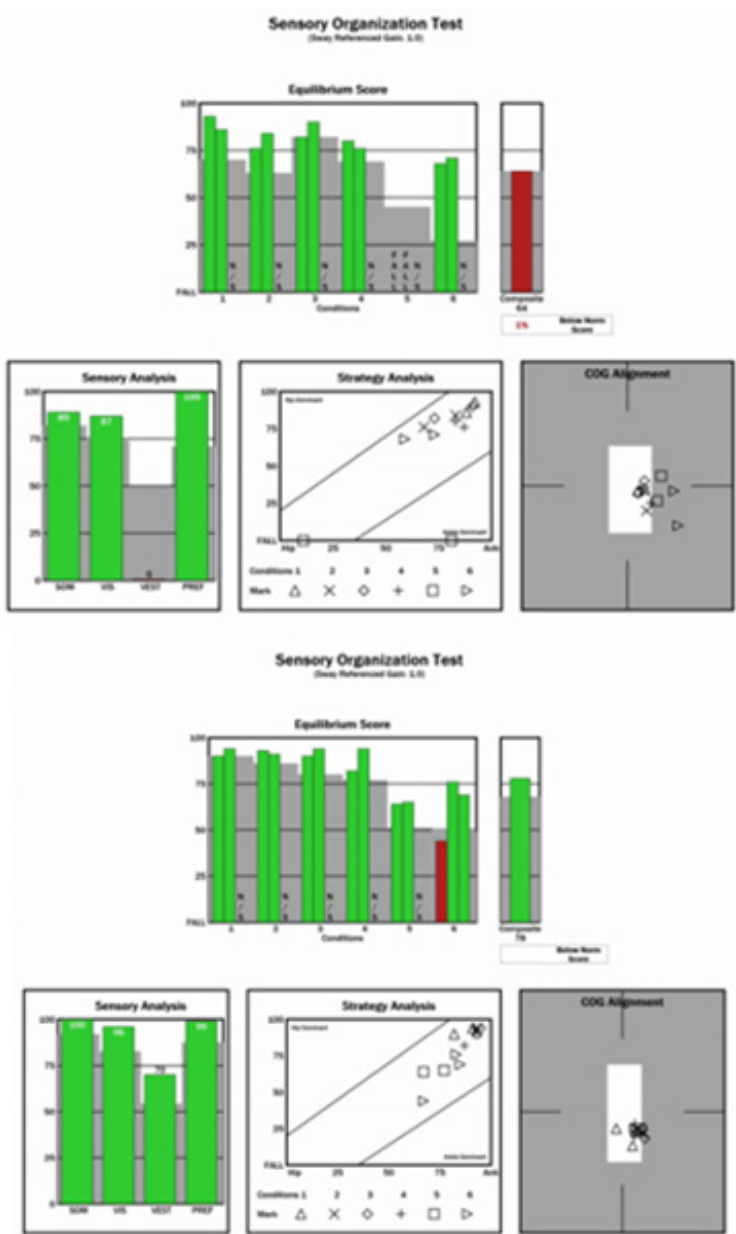

Figure I (Top) Initial evaluation Sensory Organization Test of the computerized dynamic posturography measures vestibular pattern of disequilibrium. (Bottom) Follow up posturographic data reveals a normalized pattern of postural stability indicative of central compensation. 


\section{Rotary chair}

Kinetic rotary chair testing was performed using step velocity stimulus at 100 degrees/second to evaluate mid-frequency vestibuloocular reflex function; Figure 2 VOR responses to step velocity stimulation bidirectionally revealed reduced gain in predominantly left ear driven response conditions (left per and right post). This also resulted in abnormalities and asymmetries in time constant measurements. Results may be indicative that the patient remains non-compensated at mid-frequencies of the vestibulo-ocular reflex. Post rehabilitation measurements demonstrate compensation of the mid-frequencies with stabilized gain values and borderline reduced time constants.
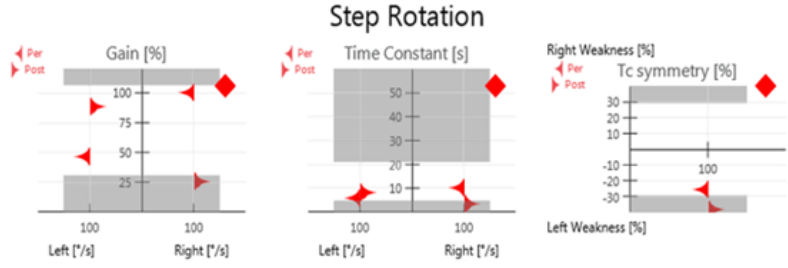

\section{Step Rotation}
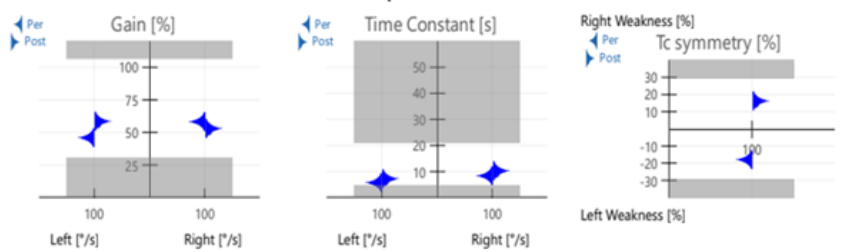

Figure 2 (Top) Step Rotation testing at initial evaluation measuring reduced gain in the left per and right post conditions. Note that these are left ear excitation conditions. Time Constants and asymmetries are also abnormal. (Bottom) Data post therapy demonstrates normalization of gain values with borderline reduced time constants. This pattern is frequently seen in patients after compensation.

\section{Video head impulse test (vHIT)}

High frequency VOR response was tested using video head impulse testing (vHIT); Figure 3. Reduced VOR gain with measured corrective saccades is seen with leftward head impulses. Rightward head thrusts revealed normal gain. This is consistent with caloric and rotational testing and indicative of non-compensated left peripheral vestibulopathy. Notably, testing at follow up reveals normalized gain values with no significant corrective saccades.

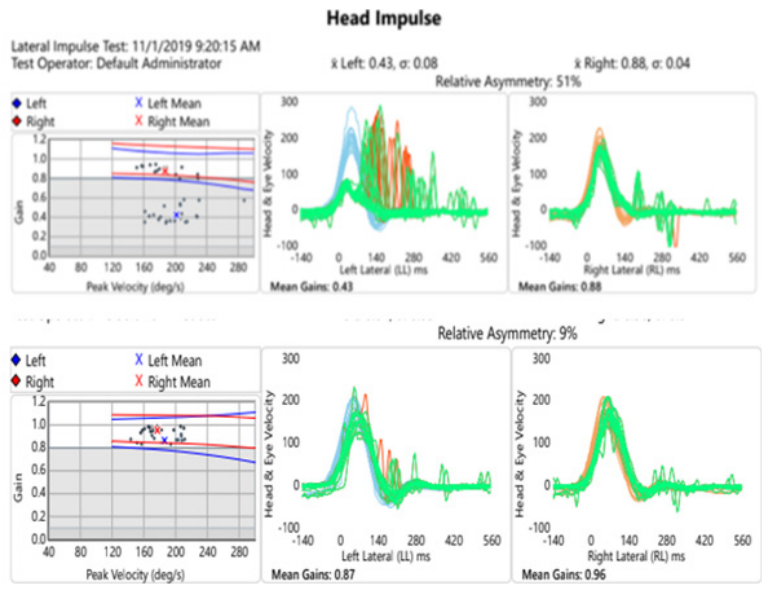

Figure 3 (Top)Video head impulse test at initial evaluation shows asymmetries with leftward head thrusts yielding significantly reduced VOR gain and corrective saccades. (Bottom) Repeat testing at follow up showed improved gain values and remission of corrective saccades.

\section{Cervical vestibular evoked myogenic potentials (cVEMP)}

Cervical vestibular evoked myogenic potential testing was performed to assess saccular function as well as the descending pathways of the inferior branch of the vestibular nerve and lower brainstem. This revealed latencies and amplitudes within normal limits for left and right ears; Figure 4.
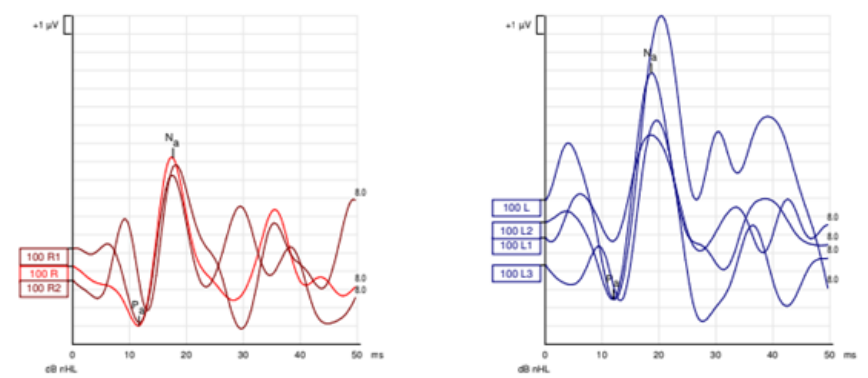

Figure 4 cVEMP recordings reveal symmetrical latency and amplitude measurements with robust waveform morphology.

\section{Dynamic visual acuity}

Complaints of visual disturbance and blurred vision was assessed with American Institute of Balance Computerized Dynamic Visual Acuity Test (AIB-CDVAT) and was positive for oscillopsia in the horizontal plane at $2 \mathrm{~Hz}$; Figure 5. Follow up AIB-CDVAT testing was performed and found to be negative for oscillopsia indicating compensation of the VOR at $2 \mathrm{~Hz}$.
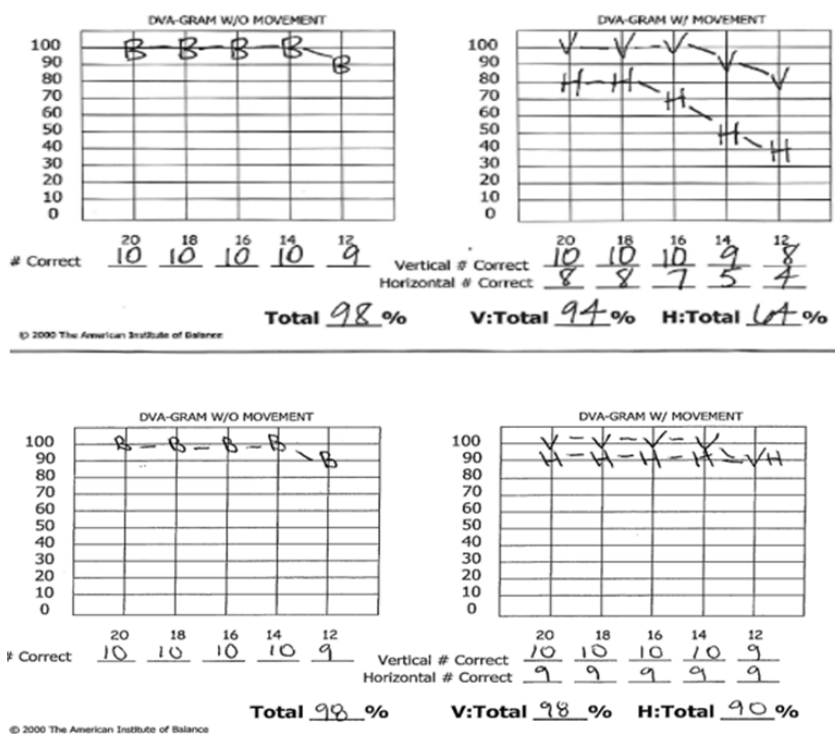

Figure 5 (Top) Initial evaluation with American Institute of Balance Computerized Dynamic Visual Acuity Test (AIB-CDVAT) shows significant decrease in visual acuity with head movement in the horizontal plane. Of note, visual acuity with vertical head movement is intact. (Bottom) Post treatment assessment of dynamic visual acuity with AIB-CDVAT shows improvement in horizontal plane. Results are now negative for clinically significant oscillopsia.

\section{Video-nystagmography (VNG)}

Results of Video-Nystagmography (VNG), revealed pendular, randomized pursuit and bi-directional optokinetic reflex testing and all within normal limits indicative of intact ocular motility. In vision denied, a spontaneous right beat nystagmus was observed. This nystagmus was direction fixed and enhanced in accordance with Alexanders Law; Figures $6 \& 7$. It also enhanced post high frequency head shake and suppressed with visual fixation. Modified Hallpike 
(mHallpike) testing revealed an upbeat rotary torsional, geotropic nystagmus which fatigued in 15 seconds in the left positioning, consisted with left ear PC-BPPV. Positional studies were otherwise unremarkable for any clinically significant nystagmus. Bithermal caloric air irrigations yielded a significant left unilateral weakness of $83 \%$. Fixation index was intact; (Figure 8) ${ }^{4-10}$

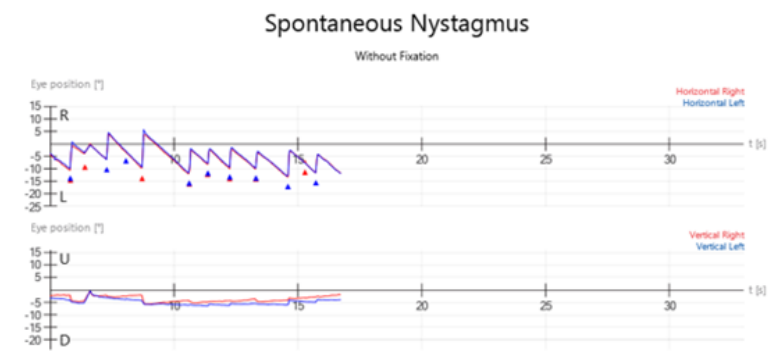

Figure 6 Right beating spontaneous nystagmus.

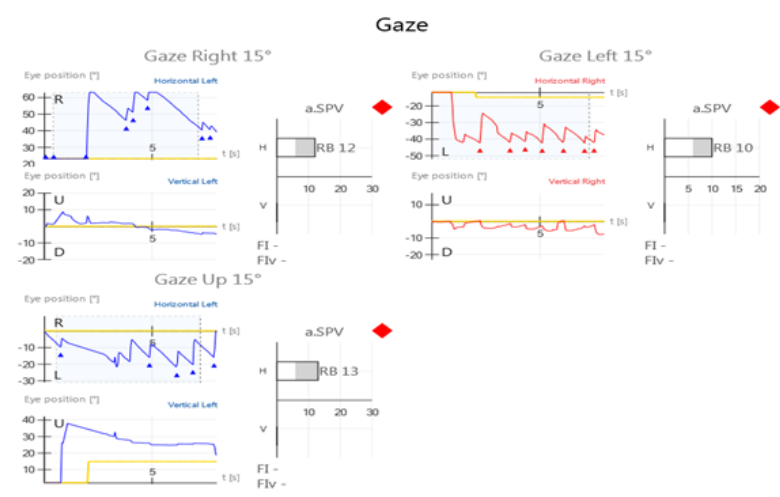

Figure 7 Direction fixed right beating nystagmus which enhances with eccentric gaze. Alexanders Law.

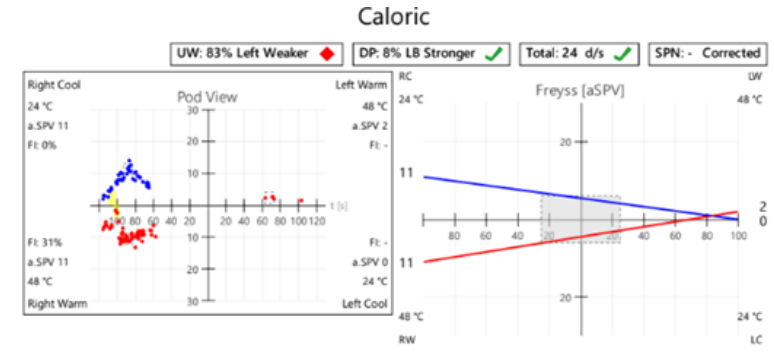

Figure 8 Left unilateral caloric weakness.

\section{Discussion}

This patient was initially diagnosed by her physician with BPPV based on a brief review of symptoms and bedside evaluation. While she did in fact have a left posterior canal BPPV, neurodiagnostic evaluation also revealed a non-compensated left unilateral vestibular dysfunction (UVD). Much like hearing loss may be conductive, due to mechanical disfunction of the tympanic membrane or ossicular chain, or sensorineural due to hair cell or auditory nerve fiber damage, the vestibular system may display similar patterns of dysfunction. Because of the mechanical nature of BPPV and sensorineural nature of the vestibular hypofunction, we will describe this case as a mixed vestibular loss. While BPPV may be the cause of her positional episodic vertigo, the identified UVD is also largely contributory to her continued functional impairment. Further, it is well documented in the literature that BPPV may occur secondary to other otologic disease. This demonstrates the need for tests of vestibular function in all patients presenting with dizziness, even those that may seem like symptoms are of BPPV etiology. Without the benefit of neurodiagnostic evaluation, this patient may have been treated for BPPV and discharged without a true assessment of vestibular function, or resolution of her functional impairment. In this case, the patient was treated successfully in one visit for the diagnosed left PC-BPPV which ameliorated episodic vertigo nearly immediately. At one week follow up she was clear of any nystagmus or subjective symptoms in modified Hallpike testing and reported full amelioration of vertigo. She did however continue to exhibit signs and symptoms consistent with a non-compensated UVD (oscillopsia, and dizziness with head movement). Being a relatively high functioning patient with no pre-existing orthopedic, neurologic, or other medical morbidities, we recommended a self-directed vestibular rehabilitation program. She was given a systematic progression of prescriptive exercises to perform at home. Protocols included gaze stabilization, adaptation, and habituation to address her functional impairments of oscillopsia and vestibular recruitment.

She returned to AIB, 14 days later, reporting significant improvement and reduction of all her symptoms. Her scores on the Dizziness Handicap Inventory (DHI) as well as objective measures including AIB-CDVAT, vHIT, Rotary Chair, and CDP all demonstrated return to normal parameters consistent with successful central compensation.

\section{Conclusion}

Neurodiagnostic tests of vestibular function play a critical part in determining etiology of symptoms, and ultimately, directing the successful management of the patient. These tests are not invasive, are timely and generally cost-effective compared to the cost of multiple visits to numerous specialists, and ultimately unnecessary laboratory and radiographic studies. It is the efficient and efficacious treatment of patients with "mixed" vestibulopathy, which reduces the challenges of daily living, lost wages, decreased social interactions, all of which may lead to anxiety and depression.

\section{Acknowledgments}

None.

\section{Conflicts of interest}

The author declares that there is no conflict of interest to disclose.

\section{Funding}

None.

\section{References}

1. Chua KW, Gans RE, Spinks SE. Demographic and clinical characteristics of BPPV patients: a retrospective large cohort study of 1599 patients. Journal of Otolaryngology-ENT Research. 2020;12(1).

2. Roberts RA, Gans RE, Montaudo RL. Efficacy of a New Treatment Maneuver for Posterior Canal Benign Paroxysmal Positional Vertigo. Journal of the American Academy of Audiology. 2006;17(8):598-604.

3. Balasteouras D, Koukoutsis G, Aspris A, et al. Benign Paroxysmal Positional Vertigo Secondary to Mild Head Trauma. Annals of Otolog, Rhinology \& Laryngology. 2017;126(1):54-60.

4. Zhai X, Liu B, Zhang Y, et al. Analysis of BPPV in patients with bone mineral density results. Lin Chung Er Bi Yan Hou Tou Jing Wai Ke Za Zhi. 2016;30(23):1865-1869.

5. Baker BJ, May C. Meniere's Disease and Concurrent BPPV in an Older Adult: A Case Report. Physical \& Occupational Therapy In Geriatrics. 2019;38(1):98-106. 
6. Kim HD, Yoon HS, Chung JH, et al. Clinical Characteristics of Traumatic Benign Paroxysmal Positional Vertigo Compared with Idiopathic Benign Paroxysmal Positional Vertigo. Korean Journal of OtorhinolaryngologyHead and Neck Surgery. 2016;59(12):819-824.

7. Bhattacharyya N, Gubbels S, Schwartz S, et al. Clinical practice guideline: benign paroxysmal positional vertigo (update). Otolaryngol Head Neck Surg. 2017;156(3_suppl):S1-S47.

8. Newman-Toker DE, Cannon LM, Stofferahn ME, et al. Imprecision in patient reports of dizziness symptom quality: a cross-sectional study conducted in an acute care setting. Mayo Clin Proc. 2007;82(11):13291340
9. Harada K, Oda M, Yamamoto M, et al. A Clinical Observation of Benign Paroxysmal Positional Vertigo BPPV after Vestibular Neuronitis VN. Acta Oto-Laryngologica. 1993;113(sup503):61-63.

10. Kroenke K, Jackson JL. Outcome in general medical patients presenting with common symptoms: a prospective study with a 2-week and a 3-month follow-up. Fam Pract. 1998;15(5):398-403. 